University of Nebraska - Lincoln

DigitalCommons@University of Nebraska - Lincoln

January 1987

\title{
Electron Correlation Effects in Nonresonant Multiphoton Ionization Processes
}

Anthony F. Starace

University of Nebraska-Lincoln, astarace1@unl.edu

Follow this and additional works at: https://digitalcommons.unl.edu/physicsstarace

Part of the Physics Commons

Starace, Anthony F., "Electron Correlation Effects in Nonresonant Multiphoton Ionization Processes" (1987). Anthony F. Starace Publications. 139.

https://digitalcommons.unl.edu/physicsstarace/139

This Article is brought to you for free and open access by the Research Papers in Physics and Astronomy at DigitalCommons@University of Nebraska - Lincoln. It has been accepted for inclusion in Anthony F. Starace Publications by an authorized administrator of DigitalCommons@University of Nebraska - Lincoln. 


\title{
Electron Correlation Effects in Nonresonant Multiphoton Ionization Processes
}

\author{
Anthony F. Starace \\ Department of Physics and Astronomy, University of Nebraska-Lincoln, Lincoln, NE 68588-0111, U.S.A.
}

\begin{abstract}
The role of electron correlations in nonresonant multiphoton processes of atoms is presented. The evidence for the validity of a lowest order perturbation theory (LOPT) approach for the laser field in the case of nonresonant processes is sketched. The growing consensus for a LOPT interpretation of even the multiply-charged ion spectra obtained with high intensity lasers is reviewed. Finally, a review of those specific electron correlation processes within LOPT that are important for the quantitative prediction of two-photon ionization cross sections is presented and some of their effects on calculated generalized cross sections are exhibited.
\end{abstract}

\section{Introduction}

Recent experiments on multiphoton ionization (MPI) [1] of rare gases and alkaline earth atoms have revealed unexpectedly intense production of multiply-charged ions by strong laser fields [2-8]. All of the experiments for xenon atoms, for example, observe the production of at least $\mathrm{Xe}^{3+}$ ions, and one group $[3,4]$ has observed the ions up to $\mathrm{Xe}^{8+}$. These results have been obtained with laser intensities in the range from $10^{12}$ $\mathrm{W} / \mathrm{cm}^{2}$ to $10^{15} \mathrm{~W} / \mathrm{cm}^{2}$ and photon energies in the range from $0.1 \mathrm{eV}$ to more than $6 \mathrm{eV}$. The number of photons absorbed necessary to produce the observed ions ranges from 2 to several hundred. Correspondingly, the energy absorbed by the atoms ranges from more than $10 \mathrm{eV}$ to several hundred $\mathrm{eV}$. Theory is only beginning to understand this unexpectedly strong nonlinear response of multielectron atoms to strong radiation fields.

Experiments clearly indicate that electron correlation effects play a fundamental role in general in MPI and in particular in explaining these new results quantitatively. As a result there is a renewed interest in theoretical descriptions of multiphoton absorption processes which go beyond the independent particle model and include the treatment of electron correlations. More specifically, one can cite at least three instances in which electron correlations need to be described: First, quantitative agreement between theoretical MPI generalized cross sections and experiment requires the inclusion of electron correlations. As is known in the theory of single photon ionization processes [9-11], neglect of electron correlations can lead to errors of as much as a factor of two. Second, multiphoton photoelectron angular distributions [12] are expected to be even more sensitive to electron correlations due to their dependence on the relative phases of transition amplitudes for alternative photoelectron channels. Third, multiphoton multiple-ionization processes require electron correlations for a correct description.

Given that electron correlations are important, theory is still faced with the decision of how to treat the laser field. In many instances, particularly for non-resonant processes, it is appro- priate to treat the laser field perturbatively. For those cases in which the lowest order of perturbation theory suffices, one may define a generalized multiphoton ionization cross section which depends exclusively on the properties of the atomic or molecular target and not on those of the laser [13]. Other instances require a non-perturbative approach. In resonant MPI, for example, the laser field may strongly couple both the initial and the resonant target states, and quantitative agreement with experiment may require detailed consideration of the laser field, including not only its maximum intensity but also its spatial, temporal, and statistical properties [14]. A similar case where non-perturbative treatments are often required is that of multiphoton autoionization [15]. As a final example of a nonperturbative regime, we note the case of laser fields intense enough to exert a greater influence on electronic motion than that of the screened Coulomb field of the nucleus. In this case it has been postulated that entire atomic subshells would undergo collective oscillations that are driven by the oscillating electric field of the laser light [4].

In this paper we review the role of electron correlations in nonresonant multiphoton processes. In Section 2 we sketch the evidence for the validity of a lowest order perturbation theory for nonresonant processes. In Section 3 we review the growing consensus for a lowest order perturbation theory interpretation of even the multiply-charged ion spectra obtained so far in Saclay, in Chicago, and in Quebec with high-intensity lasers. Finally, in Section 4 we review those specific electron correlation processes important for the quantitative prediction of two-photon ionization cross sections and exhibit some of their effects.

\section{Validity of perturbation theory for the laser field}

In lowest order perturbation theory (LOPT) the $N$-photon ionization rate, $W$, is given by [13]

$$
W=\sigma_{N} I^{N} \text {. }
$$

Here $\sigma_{N}$ is a generalized $N$-photon cross section dependent only on properties of the atomic or molecular target and on the polarization of the incident light, and $I$ is the intensity of the laser field. $W$ is usually measured in units of ions/s, $\sigma_{N^{\prime}}$ in units of $\mathrm{cm}^{2 N} \mathrm{~S}^{N-1}$, and $I$ in units of photons/(cm2 s ).

The LOPT result in Equation (1) ignores higher order perturbation terms involving absorption and emission of $S$ additional photons. The summed effect of the LOPT result in Equation (1) and such higher order terms is, of course, still the net absorption of only $N$ photons. The effect of the higher order terms on the transition rate $W$ is to introduce a dependence on higher powers of the laser intensity, $I$. 
Table I. Ionization probabilities per unit time $\left(\mathrm{s}^{-1}\right)$ for 2-, 3-, and 4-photon ionization of $\mathrm{Cs}$ and $\mathrm{K}$ at $0.53 \mu \mathrm{m}^{a, b}$

\begin{tabular}{lll}
\hline Cs & $P_{2}$ & $0.104 \times 10^{7} I^{2}-0.15 \times 10^{3} I^{3}$ \\
& $P_{3}$ & $0.795 \times 10^{2} I^{3}$ \\
& $P_{4}$ & $0.197 \times 10^{-2} I^{4}$ \\
& $P_{2}$ & $0.178 \times 10^{7} I^{2}-0.37 \times 10^{3} I^{3}$ \\
& $P_{3}$ & $0.171 \times 10^{3} I^{3}$ \\
& $P_{4}$ & $0.545 \times 10^{-2} I^{4}$
\end{tabular}

${ }^{a}$ Reprinted with permission from Aymar and Crance (1981); copyright 1981 by the Institute of Physics.

${ }^{\mathrm{b}} I$ is in $\mathrm{GW} \mathrm{cm}^{-2}$.

Aymar and Crance [16] have calculated the effect of such higher order perturbation terms on the two-photon ionization cross section of $\mathrm{Cs}$ and of $\mathrm{K}$. They also compare the magnitude of the correction terms to the LOPT result for the three and four photon ionization cross sections of these atoms. As shown in Table I, they find that such higher order corrections are insignificant up to laser powers of about $10^{11} \mathrm{~W} / \mathrm{cm}^{2}$ for the atoms considered.

For laser powers of $10^{11} \mathrm{~W} / \mathrm{cm}^{2}$ or below, then, a plot of $\log$ $W$ vs. $\log I$ should have a slope of $N$, the number of photons absorbed. Deviations from this simple behavior occur primarily near resonances, as shown in Figure 1. In this figure the slope of the $\log N_{\mathrm{i}}$ (where $N_{\mathrm{i}}$ is the number of ions produced) vs. the log I curve is shown for the 4-photon ionization of Cs process plotted vs. the detuning from the 3-photon resonant transition $6 s \rightarrow 6 f$ [17]. One sees clearly that, away from resonance, the slope is equal to 4 , whereas near resonance the slope varies rapidly between 30 and 1 . For this same 4-photon ionization process in Cs, in the neighborhood of the same $6 s \rightarrow 6 f 3$-photon resonance transition, Figure 2 shows that as the laser intensity is increased, the location of the resonance is shifted [18]. Nonresonant processes may become resonant (and vice versa) as the laser intensity is changed! In each of these instances one sees a breakdown of Equation (1) in the neighborhood of a resonance which requires a nonperturbative approach to the laser field [14, 19].

\section{Interpretation of MPI multiply-charged ion spectra}

Recent multiply-charged ion spectra obtained by the Saclay [2] and the Chicago $[3,4]$ groups using lasers with peak powers

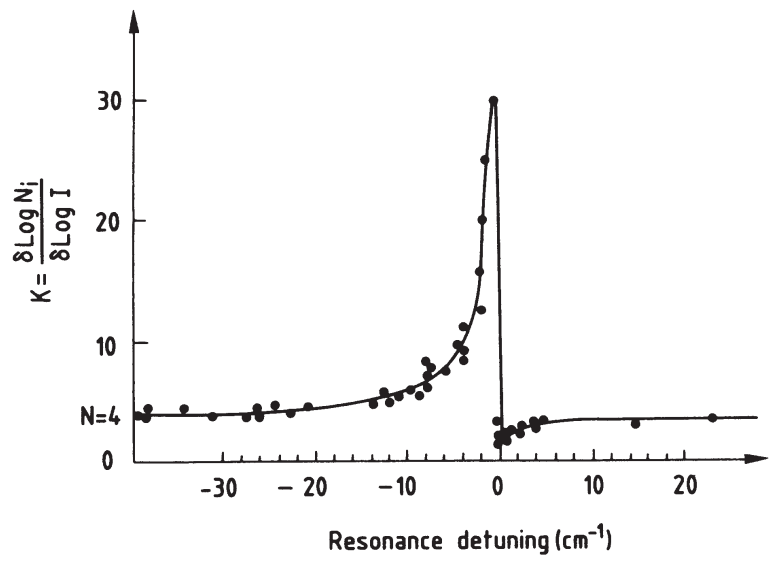

Figure 1. Variation of the slope of the $\log N_{\mathrm{i}}$ (where $N_{\mathrm{i}}$ is the number of ions) (vs. $\log I$ curve for the 4-photon ionization of Cs plotted as a function of the resonance detuning from the 3-photon $6 s \rightarrow 6 f$ transition (from Reference [17]).

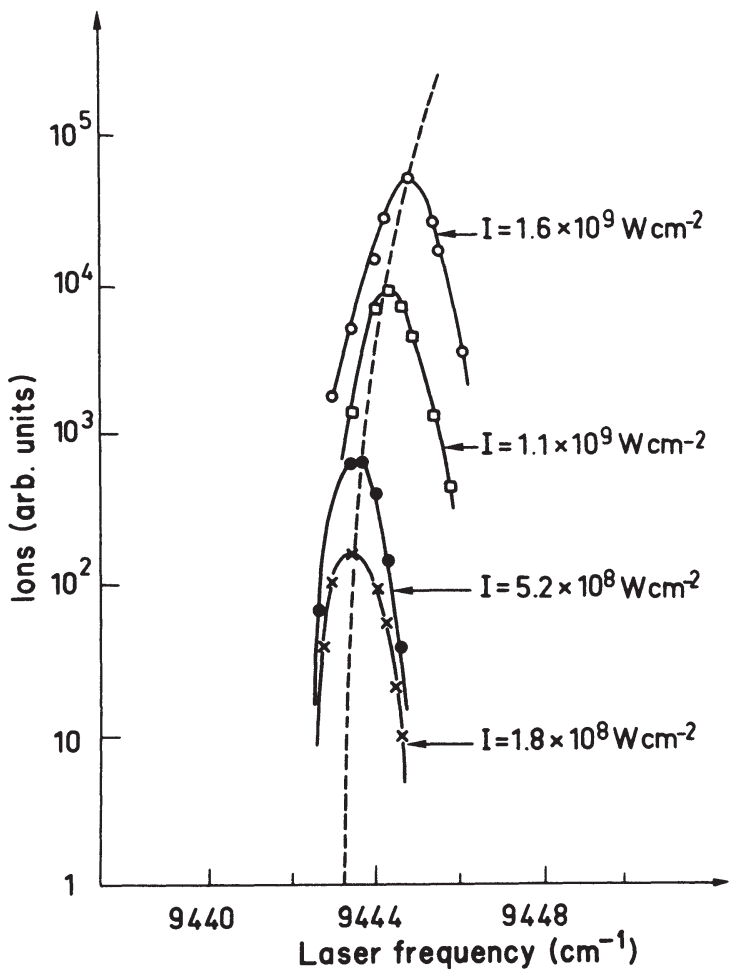

Figure 2. Variation of the number of ions in the 4-photon ionization of Cs as a function of laser frequency in the neighborhood of the resonant 3-photon transition $6 s \rightarrow 6 f$. The dashed line shows the resonance shift for increasing values of laser intensity I (from Reference [18]).

above $10^{11} \mathrm{~W} / \mathrm{cm}^{2}$ indicated such high energy absorption that the LOPT result in Equation (1) appeared to be useless for their interpretation. As shown in Figure 3 for xenon, the removal of the six outer $5 p$ electrons occurs with high probability. It appeared therefore reasonable to interpret these results as due to collective oscillations of the outer atomic subshell driven by the intense laser field [4]. Further theoretical and experimental work, however, has led to a growing consensus [20] that the multiply-charged ion spectra obtained so far may be interpreted after all using essentially the LOPT Equation (1).

\section{CHARGE STATE SPECTRA}

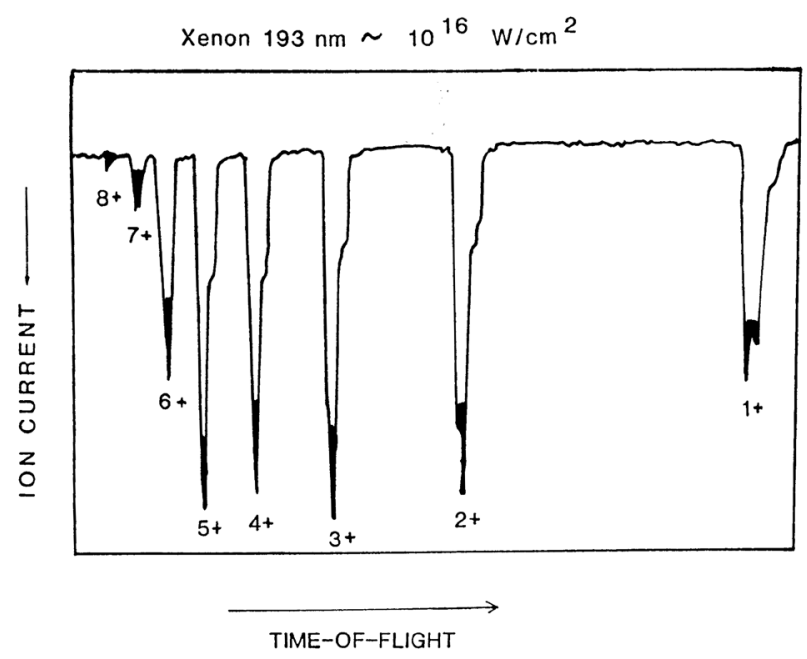

Figure 3. Charge spectra for xenon obtained by the Chicago group (from Reference [3]). 

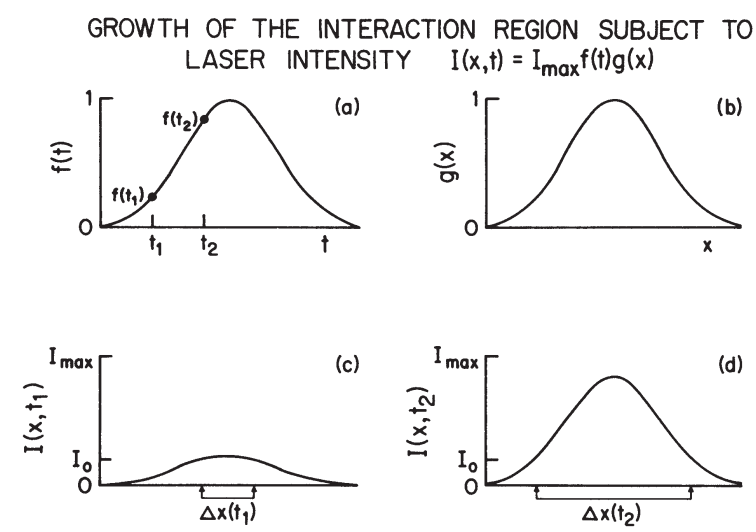

Figure 4. Schematic drawing of a laser pulse described by Gaussian distribution functions $f(t)$ and $g(x)$ for two different times, $t_{1}$ and $t_{2}$. Note how the interaction region, $\Delta x$, that experiences a laser intensity greater than $I$, increases from $\Delta x\left(t_{1}\right)$ to $\Delta x\left(t_{2}\right)$ with increasing time.

The key point, made by Lambropoulos [21], is that the spatial and temporal distribution of the laser pulse plays an essential role. In general one may describe the laser intensity by $I(\boldsymbol{r}, t)$, where

$$
I(r, t)=I_{\max } f(t) g(r)
$$

Here $f(t)$ and $g(r)$ are respectively the temporal and the spatial distribution functions of the laser pulse, each having a peak value of unity. $I_{\max }$ is the peak intensity of the laser pulse. Lambropoulos argues that as the laser pulse enters the interaction region, $f(t)$ increases from zero and, long before it reaches its peak value of unity, the effective laser intensity is large enough to completely ionize all neutral atoms in the interaction region according to Equation (1). One must realize that it takes perhaps only $10^{-13} \mathrm{~s}$ to ionize an atom [21] whereas the width of

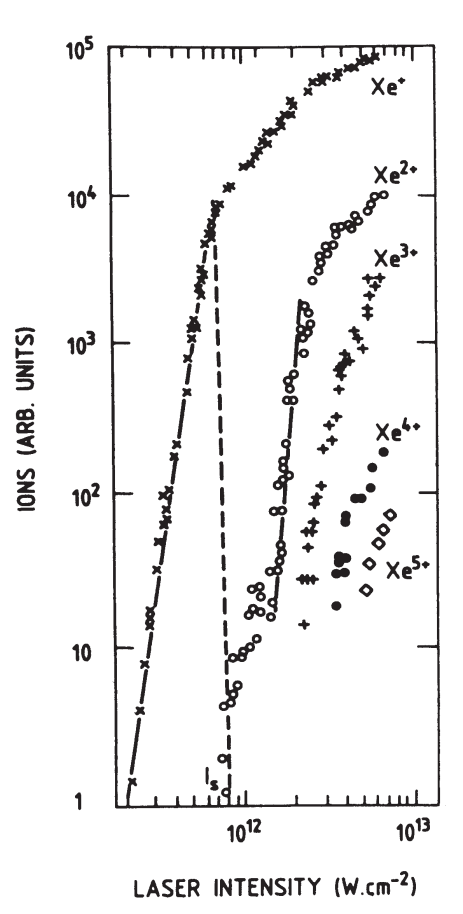

(a)

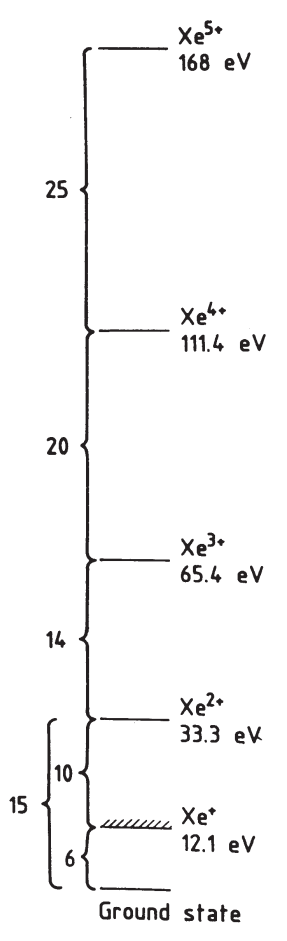

(b)
Figure 5. (a) Log-log plot of the number of ions formed in bombardment of xenon atoms with a $50-p$ sec laser pulse at $0.53 \mathrm{~m}$ as a function of laser intensity. (b) Schematic energy level diagram indicating the number of photons absorbed (from Reference [2]).

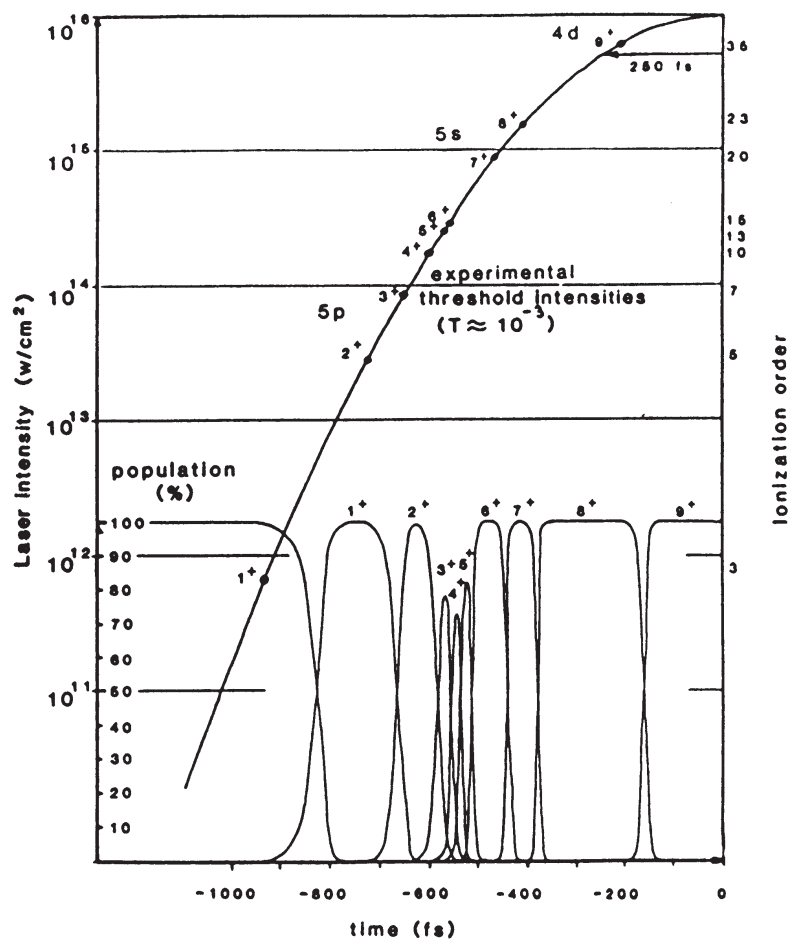

Figure 6. Xe ion population dynamics in the high intensity region of a Gaussian beam. FWHM $=500$ fs. $I_{0}=10^{16} \mathrm{~W} / \mathrm{cm}^{2}$. (From Reference [22]).

experimental laser pulses is often hundreds of times longer. Figure 4 demonstrates schematically the change in a laser pulse described by Gaussian distribution functions $f(t)$ and $g(x)$ for two times, $t_{1}$ and $t_{2}$. Once all the neutrals in the interaction region are singly ionized (i.e., once the interaction region is saturated), the singly-ionized ions are then doubly ionized, again according to Equation (1), as $f(t)$ increases further. This process continues sequentially.

Lambropoulos' sequential ionization argument [21] appears to be in accord with current experiments. Figure 5 shows the log-log plot of the ion-vs.-intensity curve of the Saclay group [2]. The various ions of xenon clearly appear to be produced sequentially as the laser intensity increases, and the slopes of the curves appear to be in accord with Equation (1). (The fact that the curves depart from linearity at the high-intensity end is interpreted as an effect of the growth of the effective interaction region with time, as demonstrated schematically in Figure 4.) Figure 6 shows clearly (for the Chicago data [22]) the temporal as well as intensity dependence of the xenon ion populations.

Further indirect confirmation of the sequential ionization interpretation of the experimental multiply-charged ion spectra is provided by two theoretical statistical analyses [23, 24]. Each distributes the laser energy among the atomic electrons statistically. The results imply sequential ionization and are in qualitative accord with experiment, thereby indicating that collective electronic behavior is unnecessary to understand the experimental results.

Quantitative agreement between theory and experiment is still lacking: the relative intensities of the multiply-charged ions produced is still much greater than expected. Wendin, Jönsson, and L'Huillier [25, 26], however, have provided a tentative explanation. They argue that the neutral atom is strongly polarized by the incident laser field, as shown schematically in Figure 7 . The result is that the effective electric field is screened 


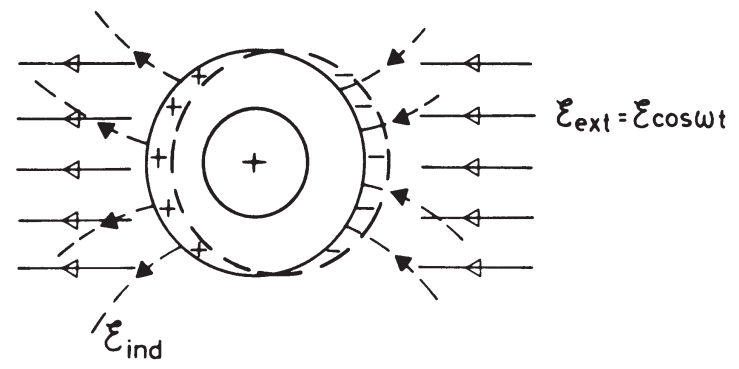

Figure 7. Polarization of an atom by an external electric field (from Reference [26]).

(i.e., reduced) within the atom, making the initial ionization stages less probable. As the electrons are sequentially ionized, however, the screening decreases, leading to a more rapid increase in the effective electric field as compared to the external electric field and hence resulting in a greater probability for ionizing the remaining outer-shell electrons.

In summary, current multiply-charged ion spectra can be largely understood qualitatively using LOPT [i.e., Equation (1)] and considering the temporal and spatial dependence of the laser pulse. Quantitative agreement between theory and experiment will require detailed consideration of electron screening effects. Experimental observation of atomic collective behavior driven by the incident laser field will require much shorter laser pulses than are currently available.

\section{Electron correlations important for two-photon ionization processes}

While the alkalis have been the focus of much theoretical attention in multiphoton studies, due to their similarity to atomic hydrogen, from the theoretical point of view, the rare gases and rare gas-like negative ions are the best candidates for developing a unified theoretical treatment of electron correlation effects on MPI processes. First, the electron correlation problem for these atoms and ions is well understood in the case of single photon ionization processes [9-11]. This understanding is of great benefit in studying this new process. Second, these atoms' spherically symmetric ground states and lack of low-lying excited states simplify the theoretical treatment. Third, the development of ArF excimer lasers has permitted the experimental measurement of the absolute two-photon ionization coefficient of xenon at $193 \mathrm{~nm}$ [27].

Only a relatively few theoretical studies of electron correlations in multiphoton ionization have been carried out. These studies have treated the rare gases and the negative hydrogen ion. Electron correlation effects on the two-photon ionization cross section of He have been treated at the level of the time-dependent Hartree-Fock approximation by Victor [28], by Ritchie [29], and by L'Huillier et al. [30]. Two-photon ionization of $\mathrm{H}^{-}$has been treated in the adiabatic hyperspherical approximation by Fink and Zoller [31] and using discrete basis set methods by Crance and Aymar [32]. While the electron correlations in these two-electron systems are significant, they are much stronger in the heavier rare gases, which are drawing increasing theoretical attention. These include the many-body perturbation theory calculation of the two-photon ionization cross section of argon of Pindzola and Kelly [33], the approximate RPA calculation of the two-photon ionization cross sections of neon and argon of Moccia, Rahman, and Rizzo [34], the transition matrix calculation of the two-photon ionization cross section for argon of Jiang and Starace [35], and the RPA

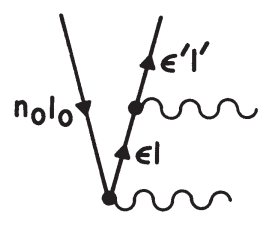

(a)

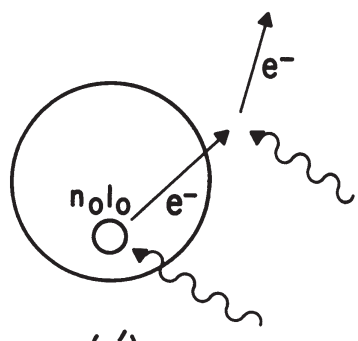

$\left(a^{\prime}\right)$
Figure 8. (a) Lowest-order (uncorrelated) many-body perturbation theory diagram for the two-photon ionization of an electron initially in the $n_{0} l_{0}$ subshell of a closed-shell atom. (a') Schematic scattering diagram for the same process.

calculation of the two-photon ionization cross section for xenon of L'Huillier and Wendin

[36].

Nearly all of the above-mentioned theoretical calculations of electron correlation treat the so-called particle-hole interactions explicitly. These are the ones that are included in the Random Phase Approximation (RPA) [37] and are the ones known to be the most important for describing single photon ionization of the rare gases [9-11]. These particle-hole interactions are strongest in the heavier rare gases. Recently L'Huillier and Wendin have shown that certain electron-scattering- type interactions are also very important in calculating the 2-photon ionization cross section of the $5 p$-subshell of xenon [36]. In what follows, we discuss each of these particle-hole and electron-scattering interactions in turn and illustrate their effects for a two-photon ionization process.

In Figure 8 we show diagrams for the lowest-order (uncorrelated) two-photon ionization process. In Figure 8(a) we have indicated the standard many-body perturbation theory diagram while in Figure 8( $\left.a^{\prime}\right)$ we present a more pictorial scattering diagram for the process

$$
\mathrm{X}+2 \gamma-\mathrm{X}^{+}\left(n_{0} l_{0}-1\right)+\mathrm{e}^{-}
$$

In Figure 8(a) the photoelectron, initially in the $n_{0} l_{0}$ subshell, is promoted by the first photon to a state with energy $\varepsilon$ and orbital angular momentum $l$ and then to a state with energy $\varepsilon^{\prime}$ and orbital angular momentum $l^{\prime}$ by the second photon. While this lowest order process does not include any electron correlation, the choice of one electron orbitals used to evaluate it will have a great influence on the relative magnitude of the higher order (i.e., correlated) diagrams. It is known from single-photon ionization studies that it is best to obtain these orbitals from a Hartree-Fock potential appropriate to the total orbital and spin angular momenta of the system; one-electron orbitals generated from average Hartree-Fock or central potentials can give single-photon ionization cross sections that differ by factors of two or more near threshold [9-11].

A second important consideration for the evaluation of the lowest order diagram in Figure 8 is the choice of resonance energies. In a two photon process one must sum over a complete set of intermediate states, both in the discrete and in the continuum region of the atom's spectrum. Because of the energy denominators in the summation, which produce strong resonance features in the calculated cross section, use of theoretical values for the resonance energies can produce quite a different spectrum from that observed experimentally. Generally it is best to use experimental values for these resonance energies [33]. 


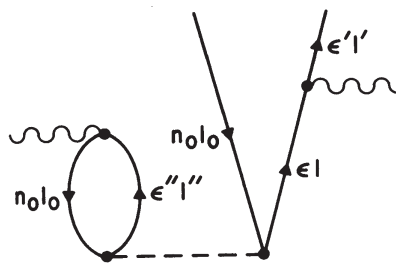

(a)

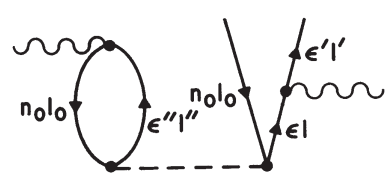

(b)

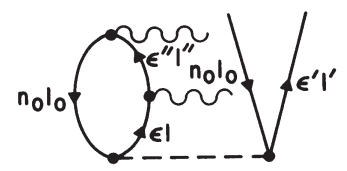

(c)

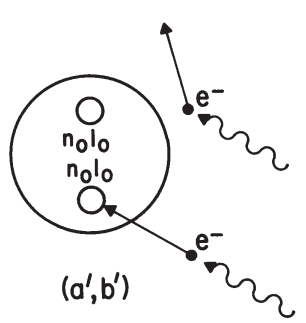

$\left(a^{\prime}, b^{\prime}\right)$

.

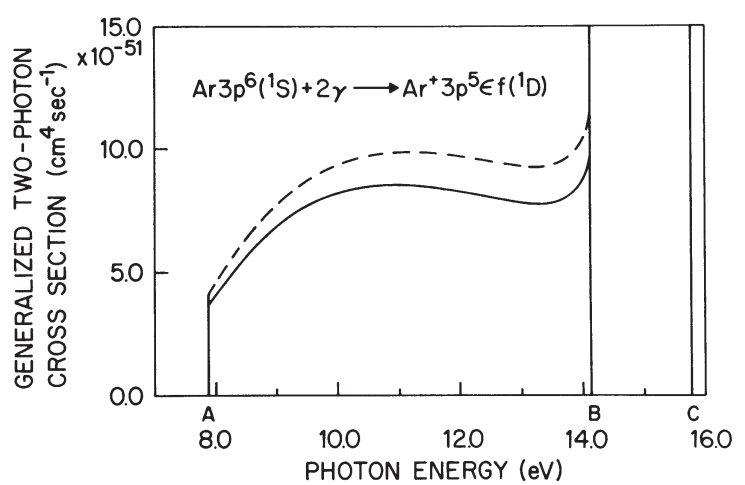

Figure 10. Transition matrix calculation of Jiang and Starace (Reference [35]) for the process. Ar $3 p^{6}\left({ }^{1} S\right)+2 \gamma \rightarrow \operatorname{Ar}^{+} 3 p^{5} \varepsilon f\left({ }^{1} D\right)$. Dashed curve $=$ Hartree-Fock calculation corresponding to the process in Figure 8 . Solid curve $=$ results obtained including all ground-state correlations shown in Figure 9.

less [35]. These results imply that in a general multiphoton process only ground state correlations which are immediately de-excited to singly-excited states by the first photon need to be considered.

Interchannel electron correlations relevant to the two-photon ionization process are shown in Figure 11. For simplicity, consider the schematic diagrams in $\left(a^{\prime}\right)$ and $\left(b^{\prime}\right)$. In ( $\left.a^{\prime}\right)$ the first photon excites an electron from subshell $n_{1} l_{1}$. While exciting the atom, the electron excites (through the Coulomb interaction) another electron out of the $n_{0} l_{0}$ subshell. The first electron is de-excited back to the $n_{1} l_{1}$ subshell, while the second electron leaves the atom, absorbing the second photon as it leaves. In $\left(b^{\prime}\right)$ the first electron absorbs the second photon before it collides with the $n_{0} l_{0}$ subshell electron. Note that diagrams similar to those in Figure 11 for which $n_{1} l_{1}=n_{0} l_{0}$ also exist; they correspond to interchannel Coulomb interactions between the alternative photoelectron channels belonging to a single subshell.

state correlation diagrams which modify the lowest-order twophoton one-electron ionization diagram in Figure 8. In Figure $9\left(a^{\prime}-c^{\prime}\right)$ we show the corresponding schematic scattering diagrams. In each case, the essential point is that the atom is considered to have initially two electrons excited out of closed shells. That is, the atom is more diffuse than in the independent-electron model used to generate the one-electron orbitals. The result is that inclusion of these interactions tends to reduce the cross sections substantially, as shown in Figure 10 for the process [35]:

$$
\operatorname{Ar}\left({ }^{1} S\right)+2 \gamma \rightarrow \operatorname{Ar}^{+}\left({ }^{2} P\right) \varepsilon f\left({ }^{1} D\right)
$$

By far the most important of the diagrams in Figure 9 is (a) [35]. This describes an atom with two electrons virtually excited in which the first photon de-excites one electron, producing a singly-excited intermediate state, and the second photon further excites the other electron. In diagram (b) the time-ordering is reversed: the first photon further excites one electron, producing a doubly-excited intermediate state, and the second photon de-excites the other electron. In diagram (c) both photons de-excite the same electron, leading again to a doubly-excited intermediate state. The schematic scattering diagrams are shown on the right hand side of the figure. [Note that $\left(a^{\prime}\right)$ and $\left(b^{\prime}\right)$ refer to the same figure]. In the case of the argon calculations shown in Figure 10, diagram (a), which has a singlyexcited intermediate state, reduces the cross sections obtained from the independent-electron model (cf. Figure 8) by $10-20 \%$ [35]. In contrast, diagrams (b) and (c), which have doubly-excited intermediate states, give corrections of the order of $2 \%$ or

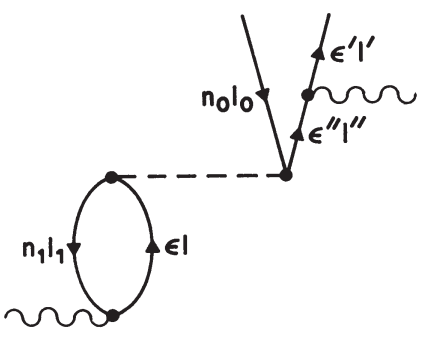

(a)

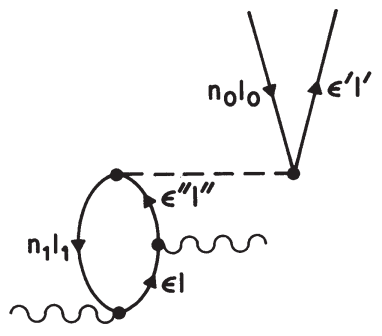

(b)
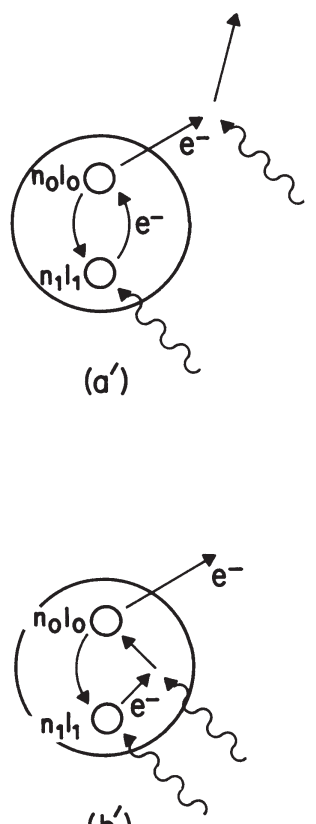

$\left(b^{\prime}\right)$
Figure 11. Interchannel interactions important for two-photon ionization processes. See text for description. Many-body perturbation theory diagrams are shown in (a) and (b). Corresponding schematic scattering diagrams are shown in $\left(a^{\prime}\right)$ and $\left(b^{\prime}\right)$. 

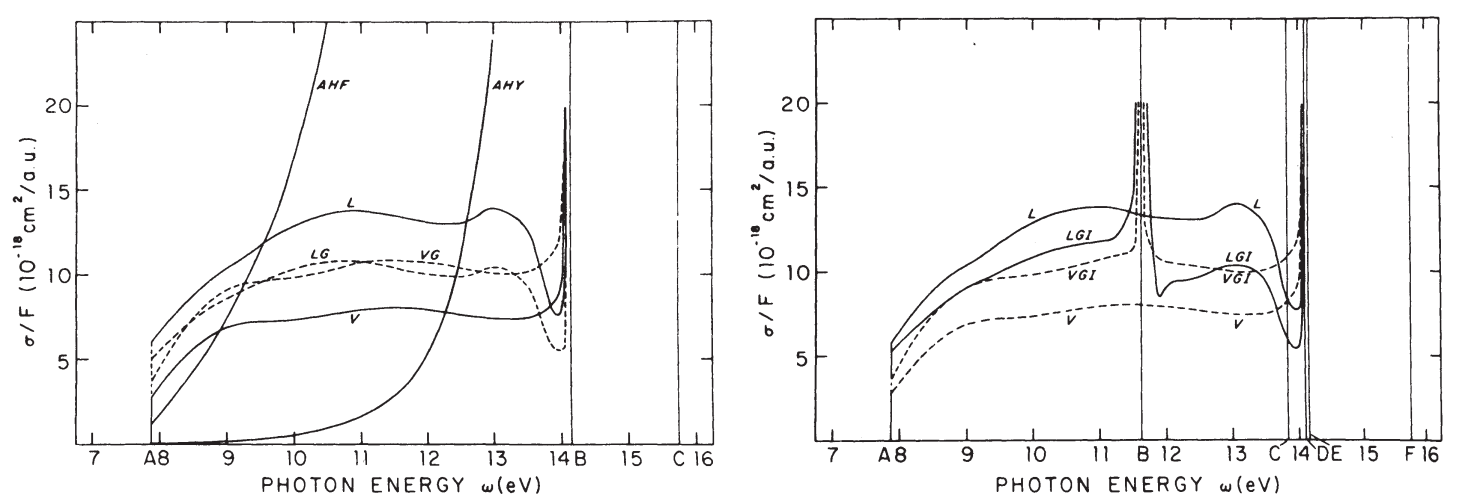

Figure 12. Many body perturbation theory calculation of Pindzola and Kelly [33] for the two-photon process, $\operatorname{Ar}+2 \gamma \rightarrow \operatorname{Ar}{ }^{+} 3 p^{5}\left({ }^{2} P\right) \varepsilon f(1 D)$. (a) LG and VG curves include ground state correlations but not interchannel interactions in length (L) and velocity (V) approximation. (b) LGI and VGI include both ground state correlations and interchannel interactions. Observe the strong $4 s\left({ }^{1} \mathrm{P}\right)$ resonance feature indicated by B that is introduced by interchannel interactions, as described in the text. (From Reference [33]).

To illustrate one effect of interchannel interactions, consider the two-photon ionization of argon shown in Equation (4). If we assume that the $\operatorname{Ar}\left({ }^{1} S\right)$ ground state is uncorrelated, then it is described in an independent electron model by Ar $1 s^{2}$ $\ldots 3 p^{6}\left({ }^{1} S\right)$. After absorption of the first photon there are two intermediate state $\left({ }^{1} P\right)$ channels:

$$
\begin{aligned}
\operatorname{Ar} 3 p^{6}\left({ }^{1} S\right)+\gamma & \rightarrow \operatorname{Ar}^{+} 3 p^{5}\left({ }^{2} P\right) \varepsilon d\left({ }^{1} P\right) \\
& \rightarrow \operatorname{Ar}^{+} 3 p^{5}\left({ }^{2} P\right) \varepsilon s\left({ }^{1} P\right)
\end{aligned}
$$

Now without interchannel interactions as in Figure 11 between the $d\left({ }^{1} P\right)$ and $s\left({ }^{1} P\right)$ channels in Equation (5), the $s\left({ }^{1} P\right)$ channel does not contribute to the $f\left({ }^{l} D\right)$ final state in (4). On the other hand, when one introduces interchannel correlations one finds [33] that the $4 s\left({ }^{1} P\right)$ resonance gives a very strong resonance feature in the $f\left({ }^{1} D\right)$ cross section, as shown in Figure 12.

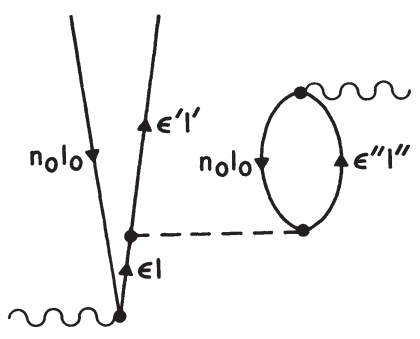

(a)

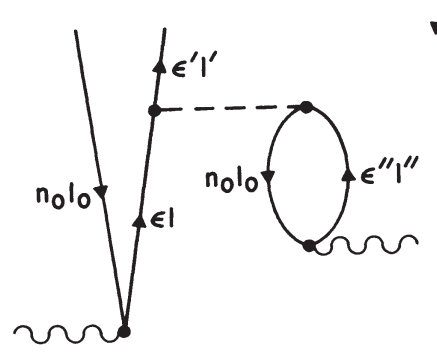

(b)

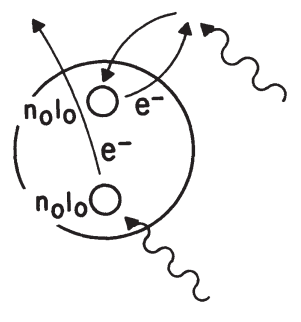

$\left(a^{\prime}\right)$

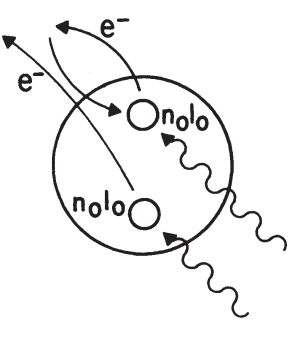

$\left(b^{\prime}\right)$
Figure 13. Electron scattering interactions relevant to 2-photon ionization processes. See text for description. Many-body perturbation theory diagrams are shown in (a) and (b). Corresponding schematic scattering diagrams are shown in $\left(a^{\prime}\right)$ and $\left(b^{\prime}\right)$.
Finally, Figure 13 shows two electron-scattering diagrams which have been evaluated for the two-photon ionization cross section of He [30] and of Xe [36]. In (a) and (a') the photoelectron produced by the first photon absorption interacts with (or scatters from) the residual ion to excite a second electron. The second photon then de-excites the second electron. In (b) and ( $\left.b^{\prime}\right)$ each absorbed photon produces an electron excited from the atomic $n_{0} l_{0}$ subshell. The first photoelectron then interacts with (or scatters from) the second photoelectron, thereby de-exciting it back to the $n_{0} l_{0}$ subshell. (An additional electron scattering diagram, not shown but very similar to (b), would have the $\varepsilon^{\prime \prime} l$ l" photoelectron produced first and the $\varepsilon l$ photoelectron second; (b') would describe this interaction as well).

The effect of these electron scattering interactions is quite significant. L'Huillier and Wendin [36] find that the electron scattering interactions increase the independent electron 2-photon cross section for the $5 p$-subshell of Xe at high photon energies by an order of magnitude! Preliminary results indicate that the electron-scattering interactions shown in Figures 13(b) and $\left(b^{\prime}\right)$ have a similarly large effect on the 2-photon ionization cross section of the $3 p$-subshell in argon [35]. In this diagram the large dipole photoionization amplitude for the outer $p^{6}$ subshells of the rare gases is contributing twice to the 2-photon transition amplitude and hence may be expected to have a large effect.

\section{Conclusions}

As both experiment and theory focus on the multiphoton ionization cross sections of the heavier rare gases, quantitative comparison of the two will require detailed treatment of electron correlations. The most important electron correlations that have been identified so far are ground-state correlations involving virtually-excited electron pairs, interchannel interactions, and electron-scattering interactions. Each of these interactions and their effects have been described here in the case where the laser intensity can be treated to lowest order of perturbation theory. As has been discussed, this case appears to be applicable to present experimental non-resonant multiphoton ionization data. Whether or not a more collective description of electron correlations will be required to understand anticipated future experimental results obtained with laser pulses having shorter times and higher intensities remains an open question. 


\section{Acknowledgments}

This work was supported in part by U.S. National Science Foundation Grant No. PHY-8601429. The hospitality of the group ER261 of the CNRS at the Observatoire de Paris, Meudon, where this manuscript was completed, is gratefully acknowledged.

\section{References}

1. For reviews of multiphoton ionization, see the various contributions in Multiphoton Ionization of Atoms (Edited by S. L. Chin and P. Lambropoulos), Academic, New York (1984).

2. L'Huillier, A., Lompré, L. A., Mainfray, G., and Manus, C., Phys. Rev. A27, 2503 (1983); J. Phys. B16, 1363 (1983).

3. Boyer, K., Egger, H., Luk, T. S., Pummer, H., and Rhodes, C. K., J. Opt. Soc. Am. B1, 3 (1994).

4. Rhodes, C. K., in Fundamentals of Laser Interactions (Edited by F. Ehlotzky), pp. 111-124, Springer, Berlin (1985); Science 220, 1345 (1985).

5. Feldmann, D., Krautwald, H. -J., and Welge, K. H., in Reference [1], pp. 223-233.

6. Kruit, P., Kimman, J., Muller, H. G., and van der Wiel, M. J., Phys. Rev. A28, 248 (1983).

7. Agostini, A., and Petite, G., in Multiphoton Processes (Edited by P. Lambropoulos and S. J. Smith), pp. 13-22, Springer, Berlin (1984).

8. Chin, S. L., Yergeau, F., and Lavigne, P., J. Phys. B18, L213 (1985); Yergeau, F., Chin, S. L., and Lavigne, P., preprint.

9. Starace, A. F., Applied Optics 19, 4051 (1980).

10. Starace, A. F., in Handbuch der Physik, Vol. 31 (Edited by W. Mehlhorn), pp. 1-121, Springer-Verlag, Berlin (1982).

11. Starace, A. F., in Fundamental Processes in Energetic Atomic Collisions (Edited by H. O. Lutz, J. S. Briggs, and H. Kleinpoppin), pp. 69-110, Plenum, New York (1983).

12. Leuchs, G., and Walter, H., in Reference [1], pp. 109-132.

13. Lambropoulos, P., Adv. At. Mol. Phys. 12, 87 (1976).

14. Crance, M., in Reference [1], pp. 65-108.
15. Lambropoulos, P., and Zoller, P., in Reference [1], pp. 189-222.

16. Aymar, M., and Crance, M., J. Phys. B14, 3585 (1981).

17. Morellec, J., Normand, D., and Petite, G., Phys. Rev. A14, 300 (1976).

18. Lompré, L. A., Mainfray, G., Manus, C., and Thebault, J., J. Phys. (Paris) 39, 610 (1978).

19. Gontier, Y., and Trahin, M., in Reference [1], pp. 35-64.

20. Robinson, A. L., Science 232, 1193 (1986).

21. Lambropoulos, P., Phys. Rev. Lett. 55, 2141 (1985).

22. Johann, U., Luk, J. S., McIntyre, I. A., McPherson, A., Schwarzenbach, A. P., Boyer, K., and Rhodes, C. K., Preprint of paper presented at the Optical Society of American Annual Meeting, 24-26 March 1986, Monterey, CA.

23. Crance, M., J. Phys. B18, L155 (1985).

24. Mu, X. D., Aberg, T., Blomberg, A., and Crasemann, B., Phys. Rev. Lett. 56, 1909 (1986).

25. Wendin, G., Jönsson, L., and L'Huillier, A., Phys. Rev. Lett. 56, 1241 (1986).

26. L'Huillier, A., Jönsson, L., and Wendin, G., Phys. Rev. A33, 3938 (1986).

27. McCown, A. W., Ediger, M. N., and Eden, J. G., Phys. Rev. A26, 3318 (1982).

28. Victor, G. A., Proc. Phys. Soc. 91, 825 (1967).

29. Ritchie, B., Phys. Rev. A16, 2080 (1977).

30. L'Huillier, A., Jönsson, L., and Wendin, G., Phys. Rev. A33, 3938 (1986).

31. Fink, M. G. J., and Zoller, P., J. Phys. B18, L373 (1985).

32. Crance, M., and Aymar, M., J. Phys. B18, 3529 (1985).

33. Pindzola, M. S., and Kelly, H. P., Phys. Rev. A11, 1543 (1975).

34. Moccia, R., Rahman, N. K., and Rizzo, A., J. Phys. B16, 2737 (1983).

35. Jiang, T. F., and Starace, A. F., Unpublished.

36. L'Huillier, A., and Wendin, G., Preprint.

37. Amusia, M. Ya., and Cherepkov, N. A., Case Studies in Atomic Physics 5, 47 (1975). 